\title{
Die Skoliose im Wachstumsalter
}

\section{P. Bernstein ${ }^{1}$, J. Seifert ${ }^{2}$}

1 UniversitätsCentrum für Orthopädie und Unfallchirurgie, Universitätsklinikum Carl Gustav Carus, Dresden

2 Chefarzt Interdisziplinäre Wirbelsäulenchirurgie, AKG Klinik Hohwald GmbH, Fachkrankenhaus für Orthopädie und Rheumaorthopädie, Neustadt/Sachsen

Die Beschäftigung der Medizin mit Wirbelsäulenverkrümmungen reicht zurück bis in die Antike. Während in der Zeit vom 16. bis 20. Jahrhundert vor allem diverse Stütz- und Korrekturapparate entwickelt wurden, begann in der Mitte des 20. Jahrhunderts die Ära der operativen Skoliosebehandlung. Die Integration des Wissens über bis heute verfeinerte konservative und operative Möglichkeiten erlaubt uns heute eine weitestgehende Kontrolle der Deformität.
Angesichts des großen Armamentariums an therapeutischen Möglichkeiten ist es bemerkenswert, dass unser Verständnis der Pathogenese noch im Dunkeln liegt. Dieser Artikel beleuchtet die wesentlichen Bausteine von Diagnostik und Therapie, wirft aber auch Licht auf aktuelle Erkenntnisse zur Pathogenese.

\section{Begriffsbestimmung}

Wir unterscheiden Skoliosen bei Kindern und Jugendlichen von Skoliosen Erwachsener. Während der Ausprägungsgrad Ersterer vom Wachstum abhängt, entstehen Skoliosen bei Erwachsenen in der Regel aufgrund von Degenerationsprozessen. Dieser Beitrag fokussiert lediglich auf die Skoliosen bei Kindern und Jugendlichen.

\section{Abkürzungen}

\begin{tabular}{|c|c|}
\hline AIS & Adoleszentenskoliose \\
\hline CrCo & Chromhexacarbonyl \\
\hline DMA & Duchenne-Muskelatrophie \\
\hline GdB & Grad der Behinderung \\
\hline ICP & infantile Zerebralparese \\
\hline NSAID & $\begin{array}{l}\text { Nonsteroidal anti-inflammatory Drug } \\
\text { (nichtsteroidales Antiphlogistikum) }\end{array}$ \\
\hline PCA & $\begin{array}{l}\text { Patient controlled Analgesia } \\
\text { (patientenkontrollierte Analgesie) }\end{array}$ \\
\hline PDK & Periduralkatheter \\
\hline RVAD & Rib vertebral Angle Difference \\
\hline SMA & spinale Muskelatrophie \\
\hline VDS & ventrale Derotationsspondylodese \\
\hline VEPTR & Vertical expandable prosthetic Titanium Rib \\
\hline
\end{tabular}

\section{Diagnostisches Vorgehen}

\section{Anamnese}

Im Rahmen der Anamneseerhebung sollte bei neurologisch gesunden Kindern auf die Familienanamnese Wert gelegt werden (s.u. Hintergrundwissen „Genetik der idiopathischen Skoliosen“). Häufig gibt es in der nächsten Verwandtschaft Angehörige, die ebenfalls eine Skoliose hatten. In der Kindesanamnese sind etwaige Operationen (z.B. bei angeborenem Herzfehler), Bestrahlungen (z.B. nach Operation eines Wilms-Tumors) am Rumpf oder Unfälle (Z.n. Wirbelfraktur) zu erfragen.

\section{Definition}

\section{Skoliose}

Mit Skoliosen werden Erkrankungen beschrieben, die bei unterschiedlicher Genese zu einer strukturellen Deformität der Wirbelsäule mit inhärenter Neigung zur Dekompensation in sagittaler, koronarer und transversaler (Rotation) Richtung führen. Innerhalb der Wirbelsäulendeformität können nichtstrukturelle Skoliosen auftreten, die als biomechanische Reaktion auf die eigentliche Strukturerkrankung gelten müssen.

Für die Erfüllung der Definition wird eine Verkrümmung in der koronaren (a.-p.) Ebene von $10^{\circ}$ nach Cobb [1] gefordert. 
Hat das Kind Schmerzen, wann treten diese auf? Die Skoliose per se ist eine schmerzlose Erkrankung. Wenn Schmerzen auftreten, insbesondere unabhängig von Belastung, muss zwingend eine weiterführende Abklärung mittels MRT zum Ausschluss einer sekundären Skoliose erfolgen

- Cave: Bei Auftreten von Schmerzen kann eine sekundäre Skoliose vorliegen.

Die Ermittlung des pubertären Reifegrades erfolgt sowohl anamnestisch (Eintritt der Menarche oder des Stimmbruchs) als auch im Verlauf klinisch (Brustwachstum, Verbreiterung des Beckens).

\section{Klinik}

Klinisch fällt die Skoliose bei der Betrachtung des entkleideten Rumpfes von hinten durch asymmetrische Taillendreiecke, Schulterasymmetrie und ggf. Lotüberhang auf. Die Rotationskomponente wird im Vorneigetest als Rippenbuckel und Lendenwulst sichtbar. Auf das Vorliegen einer Beinlängendifferenz ist zu achten.

Die Betrachtung des seitlichen Profils kann Hinweise auf begleitende Pathologien (Kyphoskoliosen, Spondylolisthesen) geben und ist für die Planung des therapeutischen Vorgehens (z. B. bei Flachrücken) wichtig.

Auf das Vorkommen von Café-au-Lait-Flecken sollte geachtet werden bzw. dies sollte hinterfragt werden, um Hinweise für das Vorliegen einer Neurofibromatose zu bekommen.

Bei neuromyopathisch kranken Kindern ist die Erfassung der funktionellen und vitalen Einschränkung durch die Grunderkrankung (z.B. reduzierte Vitalkapazität, Rollstuhl oder noch erhaltene Gehfähigkeit) und durch die Skoliose essenziell. Im Gegensatz zur idiopathischen Skoliose steht hier nicht die rotatorische Deformität, sondern die Dekompensation des Lots mit dem Herausfallen des Kindes aus dem Sitz und die Verkrümmung in der a.-p. und seitlichen Ebene im Vordergrund. Mitunter bestehen bereits sichtbare Einschränkungen in der Pflege.

\section{Bildgebende Untersuchung}

Die Basisdiagnostik wird komplettiert durch die RöntgenWirbelsäulenganzaufnahme in 2 Ebenen - idealerweise im Stehen. Für spezielle Fragestellungen in der Operationsplanung werden Bending-Aufnahmen benötigt, im Rahmen der Korsetttherapie werden Aufnahmen im

\section{Übersicht}

\section{Diagnostik der Skoliose}

- Anamnese:

- Grunderkrankung(en)

- OPs

- Radiatio

- Unfälle

- Familienanamnese

- Schmerzen

- Reifegrad:

- Menarche

- Stimmbruch

- Klinische Untersuchung:

- Taillendreiecke

- Schulterasymmetrie

- Lotüberhang

- Rippenbuckel

- Lendenwulst

- Röntgen:

- Wirbelsäule im Stehen

Korsett angefertigt. Bei Hinweisen auf eine sekundäre Skoliose oder bei auffälligen neurologischen Befunden im Rahmen der klinischen Untersuchung ist die Durchführung einer MRT wichtig.

Die verschiedenen Arten der Skoliosen bei Kindern und Jugendlichen sind im Folgenden dargestellt.

\section{Kongenitale Skoliosen}

Während der embryonalen Wirbelsäulenentwicklung kommt es entlang der Somitenachse zu Resegmentierungsprozessen, in deren Gefolge um eine Bandscheibe die jeweils benachbarten Wirbelkörperhälften aus einem primitiven Somitensegment entstehen. Einseitige Formationsfehler (Halbwirbel) können zu kongenitalen Skoliosen führen. Störungen im späteren Chondrifizierungsprozess können eine metaplastische Ossifikation des anterioren Anulus fibrosus und damit einseitige (lateral bar, Kyphoskoliosen) oder beidseitige (kongenitale Kyphose) Segmentationsstörungen zur Folge haben. Nicht selten treten Wirbelkörperfehlbildungen kombiniert auf.

Die Prognose einer kongenitalen Deformität lässt sich nicht mit letzter Sicherheit vorhersagen und muss daher im Verlauf der Entwicklung begleitet werden (halbjährlich) - mitunter sind auch mehrere therapeutische $\mathrm{Zu}$ gänge zu verschiedenen Zeiten erforderlich. 
Trotzdem lassen sich aus den morphologischen Gegebenheiten folgende Anhaltspunkte für eine Prognose formulieren:

- Halbwirbel ohne eigene Bandscheibe oder kompensierte Formationsstörungen wie Schmetterlingswirbel oder gegenüberliegende Halbwirbel sind nicht progredient.

- Isolierte Halbwirbel mit eigenen Bandscheiben (Abb. 1 a) sind in ihrem Wachstumsverhalten unsicher vorherzusagen. Eine OP-Indikation sollte bei Progredienz auf über $40^{\circ}$ und vor der Einschulung gestellt werden.

- Liegen Halbwirbel mehrere Segmente versetzt gegenüber, kann es zur hemimetameren Verschiebung und damit zur Ausbildung einer sekundären Skoliose kommen. Da sich diese Halbwirbel gegenseitig ausgleichen, ist diese Missbildung gutartig und bedarf nur selten einer Operation.

- Einseitige laterale Bar-Bildungen (Abb. 1 b) führen, abhängig von der Anzahl überbrückter Segmente, zu stetig progredienten Skoliosen mit einer Zunahme von durchschnittlich $5^{\circ} / \mathrm{J}$ ahr. Sind die Segmentationsstörungen mit kontralateralen oder kontraposterolateralen Halbwirbeln assoziiert (Abb. 1 c), kommt es zu rasch progredienten skoliotischen und kyphoskoliotischen Deformitäten. Diese Deformitäten bergen ein eigenes neurologisches Risiko durch die möglicherweise entstehende kyphotische Subluxation der Wirbelsäule. Die chirurgische Therapie durch Halbwirbelresektion oder In-situ-Fusion sollte idealerweise ab dem Alter von 2 Jahren erfolgen. Einerseits müssen die Strukturen knöchern entsprechend ausgereift sein, auf der anderen Seite soll der Entwicklung von Sekundärskoliosen möglichst wenig Chance gegeben werden.

- Gemischte Fehlbildungen, einschließlich thorakaler Fehlbildungen mit Rippenfusionen erfordern möglicherweise frühzeitig wachstumslenkende Eingriffe (z.B. VEPTR [Vertical Expandable Prosthetic Titanium Rib], Depuy Synthes).

Cave: Kinder mit kongenitalen Skoliosen sind häufig mit weiteren spinalen (Klippel-Feil), intraspinalen (Diastematomyelien, Spina bifida) Organfehlbildungen (Herz, Urogenitaltrakt) belastet.

Therapeutisches Vorgehen. Eine konservative Therapie macht bei kongenitalen Skoliosen keinen Sinn. Wenn eine Progredienz festgestellt wird, müssen operative Behandlungskonsequenzen erwogen und frühzeitig mit den Eltern besprochen werden.

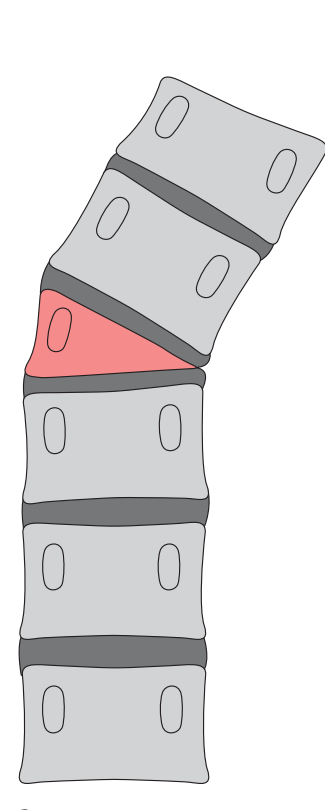

a
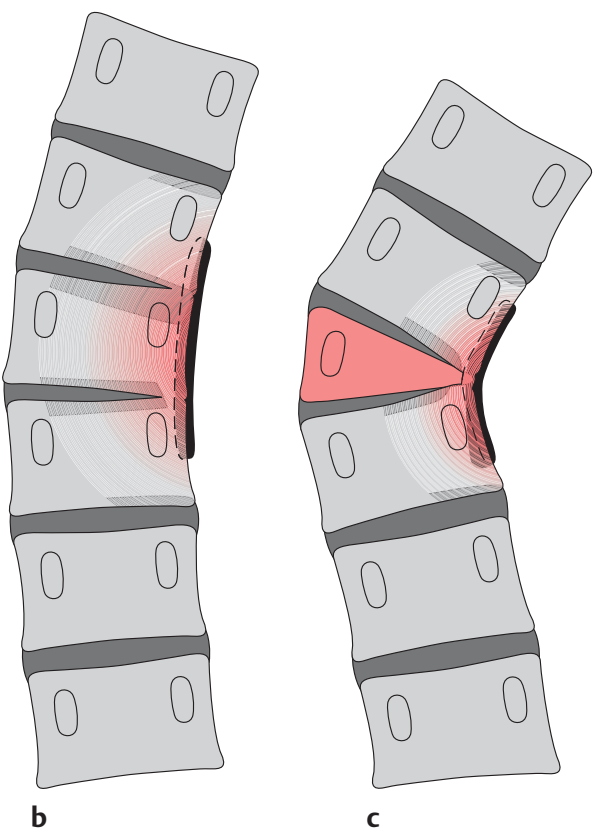

C
Abb. 1 - Schema für die wichtigsten Kategorien von koronaren Wirbelkörperfehlbildungen. a Halbwirbel (Formationsstörung). b Unilateral Bar (Segmentationsstörung). c Unilateral Bar + kontralateraler Halbwirbel (kombinierte Fehlbildung). Liegen Halbwirbel unvollständig (posterior) vor oder dehnt sich die Lateral Bar nach anterolateral aus, kommt es zusätzlich zur Kyphosenbildung.

Eine progrediente kongenitale Skoliose erfordert am ehesten eine operative Therapie.

\section{Neuromuskuläre Skoliosen}

\section{Skoliosen bei Kindern mit infantiler Zerebralparese (ICP)}

Spastische Grunderkrankungen führen mit einem größeren Risiko zu einer Skoliose als Muskelhypotonien [2]. Die Inzidenz von Skoliosen unter tetraspastisch gelähmten Kindern beträgt 64\%, je stärker die motorische Behinderung, desto größer das Risiko. Fehlt außerdem noch die Kopfkontrolle, steigt die Inzidenz auf 90\%.

Die Entwicklung einer neuromyopathischen Skoliose bei Kindern vor dem 6. Lebensjahr ist extrem selten. Üblicherweise fangen die Kinder im Alter bis 10 Jahre an, sich auf eine bevorzugte Seite zu lehnen, was als Zeichen für die Entwicklung einer flexiblen Kurve zu deuten ist. Im präpubertären Wachstumsschub werden Verschlechterungen von $2-4^{\circ} /$ Monat beobachtet. Oberhalb von $80^{\circ}$ wird die Verkrümmung steif und das aufrechte Sitzen immer mehr behindert. 
Therapeutisches Vorgehen. In der Therapieplanung müssen die Eltern frühzeitig über die Prognose und die zu erwartende Operation aufgeklärt werden. Korrigierende Rumpforthesen können den Verlauf der Erkrankung beeinflussen. Dazu müssen die Kinder jedoch frühzeitig nach den Regeln der Korsettbehandlung idiopathischer Skoliosen behandelt werden. Ein Management mittels Sitzschalenzurichtung hat lediglich supportiven Charakter.

Solange die Skoliosen im Bereich von $30^{\circ}$ bleiben, besteht ein geringes Risiko für eine Progredienz. Sobald diese Marke überschritten wird $\left(40-50^{\circ}\right)$, ist mit einer Verschlechterung zu rechnen, auch wenn es sich um skelettal ausgereifte Patienten handelt. Sind die Kinder zu dem Zeitpunkt noch zu jung für eine Versteifung ( $<10$ Jahre), kommen ggf. Etappenverfahren und nichtversteifende Verfahren zum Einsatz (VEPTR, Shilla [kurzstreckige Fusion des Scheitels und langstreckige gleitende Schienung an Pedikelschrauben]).

Große Wirbelsäulenoperationen bei ICP-Patienten sind im Vergleich zu neurologisch gesunden Kindern mit einer erhöhten Morbidität belastet und sollten nur in Zentren mit einer pädiatrischen Intensivmedizin durchgeführt werden.

\section{Duchenne-Muskelatrophie, spinale Muskelatrophie}

Kommt es im Krankheitsverlauf der Duchenne-Muskelatrophie (DMA) zur Rollstuhlpflichtigkeit, entwickeln bis über 90\% der Patienten eine Skoliose [3]. Es wurde ein klarer Zusammenhang von Alter und Abnahme der Vitalkapazität und dem Ausmaß der Skoliose gezeigt. Nahezu alle Kinder mit spinaler Muskelatrophie (SMA) entwickeln eine Skoliose.

Therapeutisches Vorgehen. Die Therapie sollte frühzeitig operativ durch eine dorsale Skolioseaufrichtung (langstreckig von der oberen BWS bis L5, ggf. Becken) erfolgen, da die konservative Behandlung mittels Korsett die Winkelprogredienz nicht aufzuhalten vermag und zusätzlich die Lungenfunktion beeinträchtigt. Einige Autoren propagieren eine definitive Fusion bereits ab Winkelgraden unter $40^{\circ}$. Meist haben die Kinder zu diesem Zeitpunkt noch eine bessere Lungenfunktion, die Vitalkapazität nimmt durch die OP kurzfristig ab. Wir haben operative Maßnahmen bei einer präoperativen Reduktion der Vitalkapazität unter 30\% nicht mehr durchgeführt. Die Datenlage zur Beeinflussung der Lungenfunktion im Langzeitverlauf ist jedoch heterogen.

\section{Übersicht}

\section{Neuromuskuläre Skoliosen \\ - häufig progredient und dann frühzeitig operativ zu versorgen \\ - langstreckige dorsale Verfahren von der oberen BWS bis LWK 5 \\ - einsteifende Verfahren erst ab dem 10. Lebensjahr \\ - Einschränkungen der Vitalkapazität beachten}

Gegen eine zu frühe OP spricht der Fakt, dass die Gehfähigkeit unter der langstreckigen Fusion leidet, sodass die Indikation nur bei rollstuhlpflichtigen Patienten gestellt werden sollte.

\section{Andere Skoliosen}

Es existiert noch eine Vielzahl weiterer neurologischer Krankheitsbilder, die mit der Ausbildung einer Skoliose im Wachstumsalter einhergehen.

\section{- Myelomeningozele}

Kinder mit einer Myelomeningozele sind, sofern die Lähmung im thorakalen Bereich liegt, regelhaft von einer Skolioseentwicklung betroffen. Neben den Problemen, die ein eventuell noch vorhandenes Tethering des Rückenmarks mit sich bringen kann, bestehen die Schwierigkeiten in der knöchernen Implantatverankerung und weichteiligen Deckung, insbesondere der kaudalen Instrumentierungsanteile.

Sind die Kinder jünger als 10 Jahre, ist mitunter die Platzierung eines nachstellbaren Implantates wie des VEPTR sinnvoll. Diese Implantate erfordern wiederholte Nachstelloperationen und sind mit einer hohen Komplikationsquote (Ausbrechen, Infekte) belastet.

\section{- Neurofibromastose}

Kinder mit einer Neurofibromastose Typ I haben kurzbogig anguläre Skoliosen, die in der Regel durch eine begleitende Kyphose mit keilförmig deformierten Wirbelkörpern gekennzeichnet sind. Begleitende intraspinale Malformationen müssen vor der eigentlichen Korrektur abgeklärt werden. 


\section{Idiopathische Skoliosen}

Innerhalb der idiopathischen Skoliosen wird eine Unterteilung nach Altersgruppen beim Auftreten erster Symptome gemacht.

\section{Infantile Skoliose}

Infantile Skoliosen ( 0 - 3 Jahre) zeigen eine Prävalenz von $1 \%$ in der Bevölkerung, wobei die Hälfte der Fälle eine spontane Regression aufweist und damit eher einer skoliotischen Fehlhaltung entspricht.

Für die Prognose entscheidend ist der sogenannte MehtaWinkel (RVAD, Rib vertebral Angle Difference). Bei einem Winkel von $>20^{\circ}$ ist in $85 \%$ der Fälle mit einer Progredienz zu rechnen (s. Abb. 2).

\section{Juvenile Skoliose}

Juvenile Skoliosen treten im Alter von 4-10 Jahren auf. Sie sind häufig progredient und mit einer erhöhten OP-Quote assoziiert.

\section{Adoleszentenskoliose}

Die Adoleszentenskoliose (AIS, 11 - 18 Jahre) macht etwa $90 \%$ aller idiopathischen Skoliosefälle aus. Die Prävalenz schwankt zwischen $0,47 \%$ und 5,2\% abhängig von der ethnischen Zugehörigkeit, der Präzision der Diagnosestellung und besonders vom Geschlecht. Sind Mädchen bei milden Skoliosen $\left(11-20^{\circ}\right)$ mit einem $\mathrm{w} / \mathrm{m}$-Verhältnis von 1,4:1 nur moderat vermehrt betroffen, so steigt dieses Verhältnis bei schweren Skoliosen $\left(>40^{\circ}\right)$ auf $7,2: 1$ an [4].

\section{- Pathogenese der idiopathischen Skoliose}

Kinder mit einer idiopathischen Skoliose sind in der Regel mental und körperlich gesund. In klinischen Untersuchungen konnte jedoch eine Konkordanz mit diskreten Abnormalitäten gegenüber Nicht-Skoliose-Kindern festgestellt werden, die das Augenmerk auf pathogeneseassoziierte Organsysteme lenken. Auf neuromuskulärer Seite haben AIS-Kinder eine verschlechterte Gangkontrolle, eine dünnere rechtsseitige Großhirnrinde und kleinere Kleinhirnregionen. Andere neuromorphologische Aspekte wie die Höhe des Konusstandes, die Korrelation von Wirbelkörperhöhe und Rückenmarklänge, Muskelspindelfunktion, Corpus-callosum-Volumen oder die elektromyografische Aktivität der Rückenmuskeln

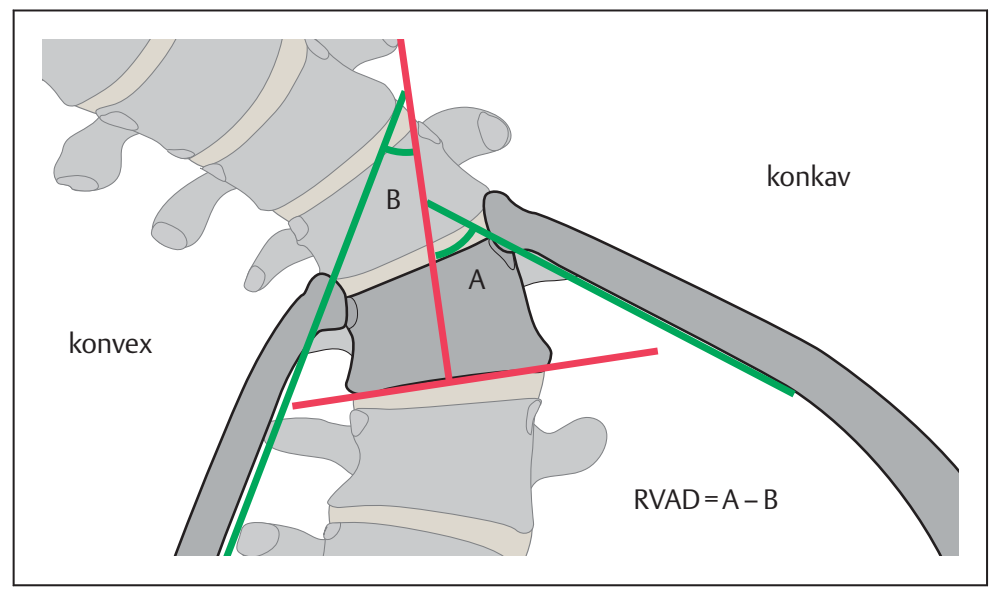

Abb. 2 - Mehta-Winkel (RVAD, Rib vertebral Angle Difference). Ein Winkel von $>20^{\circ}$ spricht für eine ungünstige Prognose einer infantilen Skoliose (aus [6]).

zeigen entweder keine oder nur schwach belegte Assoziationen mit einer Skoliose.

Aus metabolischer Sicht haben AIS-Kinder eine schlechtere Knochenqualität bei verminderter Knochendichte und geringerer Kalziumaufnahme. Anthropometrische Auffälligkeiten umfassen ein vermindertes Körpergewicht und größere Körper (wenn die Skoliose rechnerisch korrigiert wurde) sowie eine erhöhte Brustasymmetrie.

So wenig wie eine Kausalität zwischen den beobachteten Auffälligkeiten hergestellt werden kann, so schwierig gestaltet sich die experimentelle Erforschung der Skoliosepathogenese. Da die Skoliose nur beim Menschen auftritt, gibt es keine natürlichen Tiermodelle. Sekundär auftretende Skoliosen bei hypophosphatatischen Mäusen legen eine Fehlfunktion des Osteopontin - CD44 - Pfades in der knöchernen Endstrecke nahe und könnten die Konkordanz mit der schlechteren Knochenqualität erklären. Pinealektomierte Hühnchen entwickeln zu 20\% eine Skoliose. Melatoninauffälligkeiten konnten jedoch bei Skoliosepatienten nicht beobachtet werden - ebenso wenig konnte die initial vermutete genetische Auffälligkeit der Melatoninrezeptoren bestätigt werden. Es wurde aber eine asymmetrische Verteilung seines Second Messenger Calmodulin in der Rückenmuskulatur gemessen. Gestützt werden diese Erkenntnisse durch die Identifikation von Genpolymorphismen des Calmodulin-1-Gens in Skoliosepatienten. Weitere Kandidaten bislang noch kontrovers diskutierter Stoffwechselwege sind Leptin und Östrogen.

Der Schlüssel zur Pathogenese könnte im Zusammenführen dieser Erkenntnisse mit dem Roth-Porter-Konzept 
Tabelle 1

Mögliche AIS-Kandidatengene.

\begin{tabular}{|c|c|}
\hline $\begin{array}{l}\text { Genmutationen/ } \\
\text {-polymorphismen }\end{array}$ & biologische Funktion \\
\hline \multirow[t]{3}{*}{ VANGL1 } & axiale Entwicklung \\
\hline & Entwicklung des Neuralrohrs \\
\hline & Rückenmarkorientierung \\
\hline Region bei LBX1 & $\begin{array}{l}\text { Homeobox-Gen, Maus: Schlüsselregulator der Muskelvor- } \\
\text { läuferzellmigration und zuständig für die dorsale Identität } \\
\text { der Muskeln der oberen Extremität }\end{array}$ \\
\hline IL-17RC & Interleukin-17-Rezeptor in nicht hämatopoetischen Zellen \\
\hline \multirow[t]{2}{*}{ MATN1 } & Matrilin 1, Protein der Knorpelmatrix \\
\hline & $\begin{array}{l}\text { Assoziation mit Skoliose hauptsächlich in der asiatischen } \\
\text { Population }\end{array}$ \\
\hline CALM1 & Calmodulin 1, kalziumbindendes Protein \\
\hline \multicolumn{2}{|c|}{ Veränderte Genexpression } \\
\hline $\begin{array}{l}\text { Osteoblastenkultur } \\
\text { von Wirbelkörpern } \\
\text { von Skoliosepatienten } \\
\text { vs. Traumapatienten } \\
\text { Homeobox-Gene } \\
\text { (HOXB8, HOXB7, } \\
\text { HOXA13, HOXA10) }\end{array}$ & $\begin{array}{l}\text { Die Identifikation verschiedener differenziell regulierter Gene } \\
\text { lässt Rückschlüsse auf die an der Entwicklung des Krankheits- } \\
\text { prozesses beteiligten zellulären Signalwege zu. Dies beinhaltet } \\
\text { die Steuerung der Körpersegmentierung durch Homeobox- } \\
\text { Gene, die Steuerung der Knorpel-Knochen-Entwicklung } \\
\text { (COMP) und die Steuerung der Rechts-links-Differenzierung } \\
\text { und Hypophysenentwicklung (PITX1). }\end{array}$ \\
\hline \multicolumn{2}{|l|}{$\mathrm{ZIC2}$} \\
\hline \multicolumn{2}{|l|}{ FAM101A } \\
\hline \multicolumn{2}{|l|}{ COMP } \\
\hline PITX1 & \\
\hline
\end{tabular}

des dysproportionierten Wachstums von Wirbelkörpern und Rückenmark liegen. Die Beobachtung, dass die Rotationsdeformität immer aus einer relativen Lordose resultiert, wurde bereits im Jahr 1952 von Somerville gemacht. Nahezu alle AIS-Wirbelsäulen zeigen ein viel zu starkes Wachstum der Wirbelkörper im Gegensatz zu den dorsalen Elementen, einschließlich Rückenmark, was lordogen und skoliogen wirkt (Abb. 3) [8]. Die neurobiologischen Grundlagen der vermuteten neuroossären Entkopplung werden möglicherweise in den nächsten Jahren mit der Entschlüsselung des humanen „Connectoms“ zutage treten.
Hintergrundwissen

\section{Genetik der idiopathischen Skoliosen}

Bei fast allen Patienten (97\%) mit einer Adoleszentenskoliose (AIS) lässt sich eine positive Familienanamnese erheben. In der anderen Richtung besteht jedoch nur ein $11 \%$ iges AIS-Risiko für Verwandte I. Grades, während dies rapide auf $2,4 \%$ und $1,4 \%$ bei II.- und III.-gradigen Verwandten absinkt. Dies lässt den Schluss zu, dass mehrere Gene mit unvollständiger Penetranz und Expressivität ätiologisch beteiligt sind.

In den letzten Jahren sind verschiedene AIS-Kandidatengene identifiziert worden, viele konnten jedoch in Validierungsstudien nicht bestehen. Tab. 1 fasst die derzeit am ehesten infrage kommenden Gene zusammen [7] (weitere Literatur beim Autor).

Die Vielzahl der möglicherweise am Erkrankungsprozess beteiligten Gene und ein hoher Anteil sporadischer Fälle erschweren die Identifikation krankmachender Gene. Genom-weite Assoziierungsstudien stellen eine Weiterentwicklung genetischer Tests dar und haben z. B. die LBX1Region als eine relevante Schlüsselregion bei idiopathischen Skoliosen im asiatischen Raum identifizieren können. Die Erforschung epigenetischer Phänomene auf Umweltfaktoren und Hormonachsen (im Falle der AIS insbesondere Leptin und Östrogen) steht erst am Anfang. Hier ist in den nächsten Jahren mit ersten Ergebnissen zu rechnen.

\section{natürlicher Verlauf der idiopathischen Adoleszentenskoliose}

Als Faustregel lässt sich sagen, dass bei einer äußerlich im Vorneigetest deutlich sichtbaren Skoliose (etwa ab $20^{\circ}$ Cobb-Winkel; Bestimmung s. Abb. 4) etwa 3/4 der skelettal unreifen Patienten (Risser-Stadium 0-I, s.Abb. 5) einen progredienten Verlauf nehmen werden.

Je ausgeprägter die Krümmung, desto höher ist das Risiko für eine Progredienz der Skoliose (s. Abb. 6).

Das Risiko nimmt mit der skelettalen Reife ab und beträgt für das Risser-Stadium >II nur noch etwa $1 / 4$. Nichtsdestotrotz bleibt die Progredienzbeurteilung mit Unsicherheit belastet und erfordert eine wiederholte klinische und/oder radiologische Kontrolle der Patienten.

Im Langzeitverlauf (> 40 Jahre Beobachtung) besteht beim natürlichen Verlauf der idiopathischen Adoleszentenskoliose im Gegensatz zur juvenilen und kongenitalen 


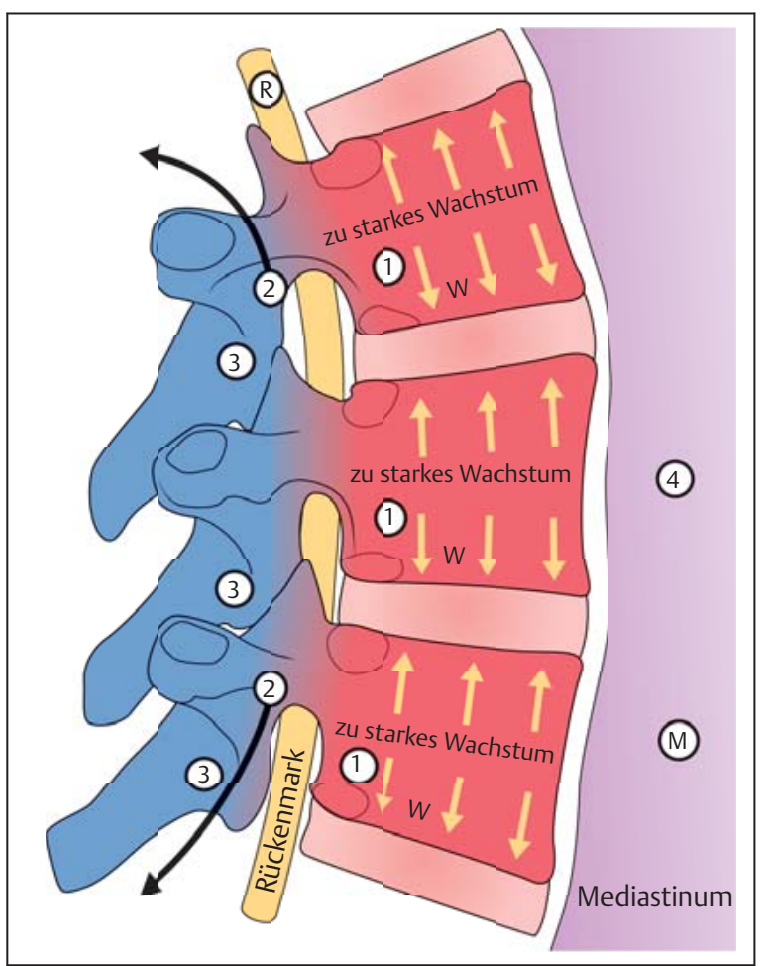

Abb. 3 - Das biomechanische Entwicklungskonzept der Skoliose [8]. (1) Ein zu starkes Wachstum des enchondral ossifizierenden Wirbelkörpers (W) führt zu einem lordosierenden Effekt. Sowohl das Rückenmark (R) als auch die perimembranöse Ossifikation der Pedikel können diesem Wachstum nicht folgen. (3) Die zunehmende Lordosierung führt zu Scherkräften und Abnahme der rotatorischen Stellungskontrolle durch die Facettengelenke. (4) Durch die asymmetrische Konfiguration des Mediastinums (M) erhält die primär sagittale Deformität eine rotatorische Komponente. Bedingt durch das Hueter-Volkmann-Gesetz können sekundäre Wirbelkörperdeformitäten entstehen (rot = vermehrtes Längenwachstum; blau = normales oder vermindertes Längenwachstum).

Skoliose keine erhöhte Mortalität [6]. Hochgradige thorakale Skoliosen (>80 Cobb-Winkel) können jedoch zu einer zusätzlichen Beeinträchtigung einer möglicherweise durch andere Faktoren (natürliche Alterung, Rauchen, Asthma) reduzierten Lungenfunktion führen. Bei Skoliosen mit einem Cobb-Winkel $>50^{\circ}$ ist mit einer jährlichen Progredienz von $0,4-1^{\circ}$ zu rechnen. Rückenschmerzen treten bei erwachsenen AIS-Patienten häufiger als in der Normalbevölkerung auf.

Die Progredienzwahrscheinlichkeit hängt vom Alter und Winkel ab.

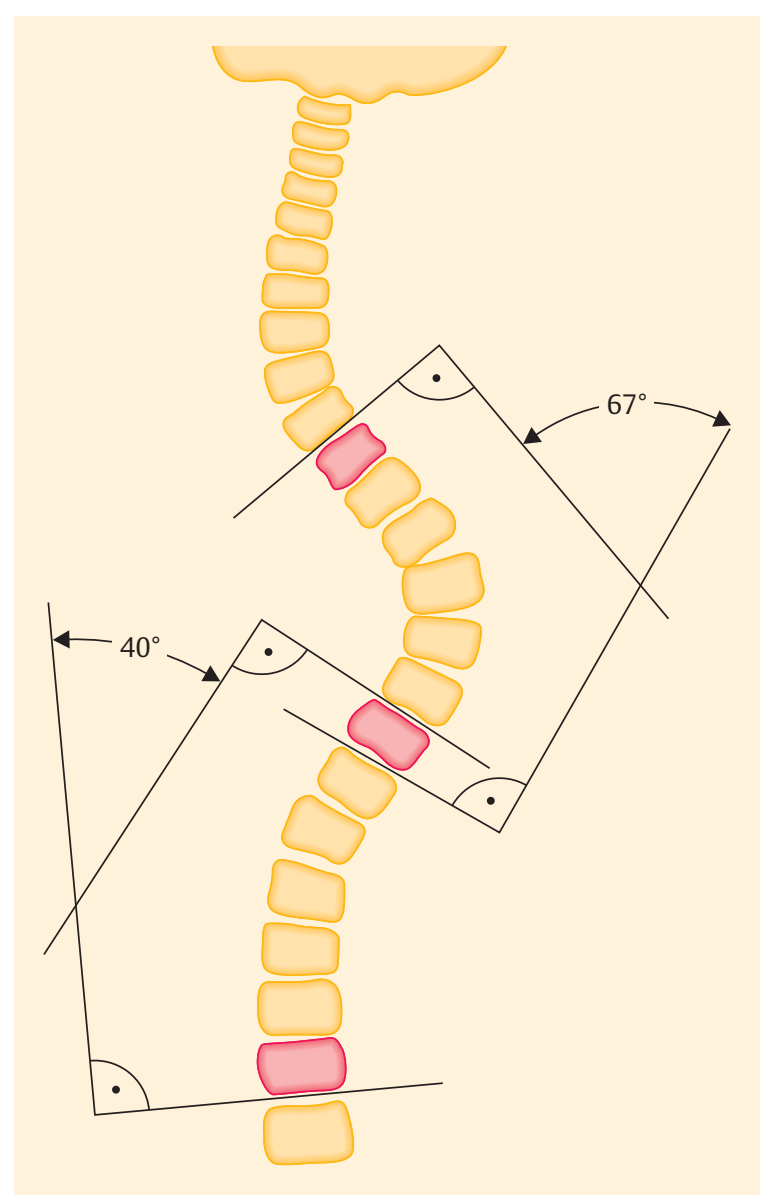

Abb. 4 - Bestimmung des Skoliosewinkels nach Cobb [1]. Die Wirbel der Kurvendurchgänge werden Neutralwirbel genannt, da sie eine neutrale Rotation aufweisen. Sie stellen die Begrenzung für das Einzeichnen des Cobb-Winkels dar (aus [11]).

\section{Therapeutisches Vorgehen}

Bei Skoliosen mit einem Cobb-Winkel $<20^{\circ}$ wird Physiotherapie verordnet und das Kind engmaschig kontrolliert. Zeigt sich hier eine deutliche Progredienz, besteht die Indikation zur Korsetttherapie.

In Abb. 7 ist der sog. Dresdner Skoliosepfad dargestellt. Die Entscheidung zur OP ist ab einem Cobb-Winkel von $40^{\circ}$ (lumbal) $/ 50^{\circ}$ (thorakal) möglich. Handelt es sich jedoch um sehr junge Patienten mit hoher Wachstumsreserve, kann auch in diesem Winkelbereich ein Korsettversuch unternommen werden. 


\section{Wirbelsäule}

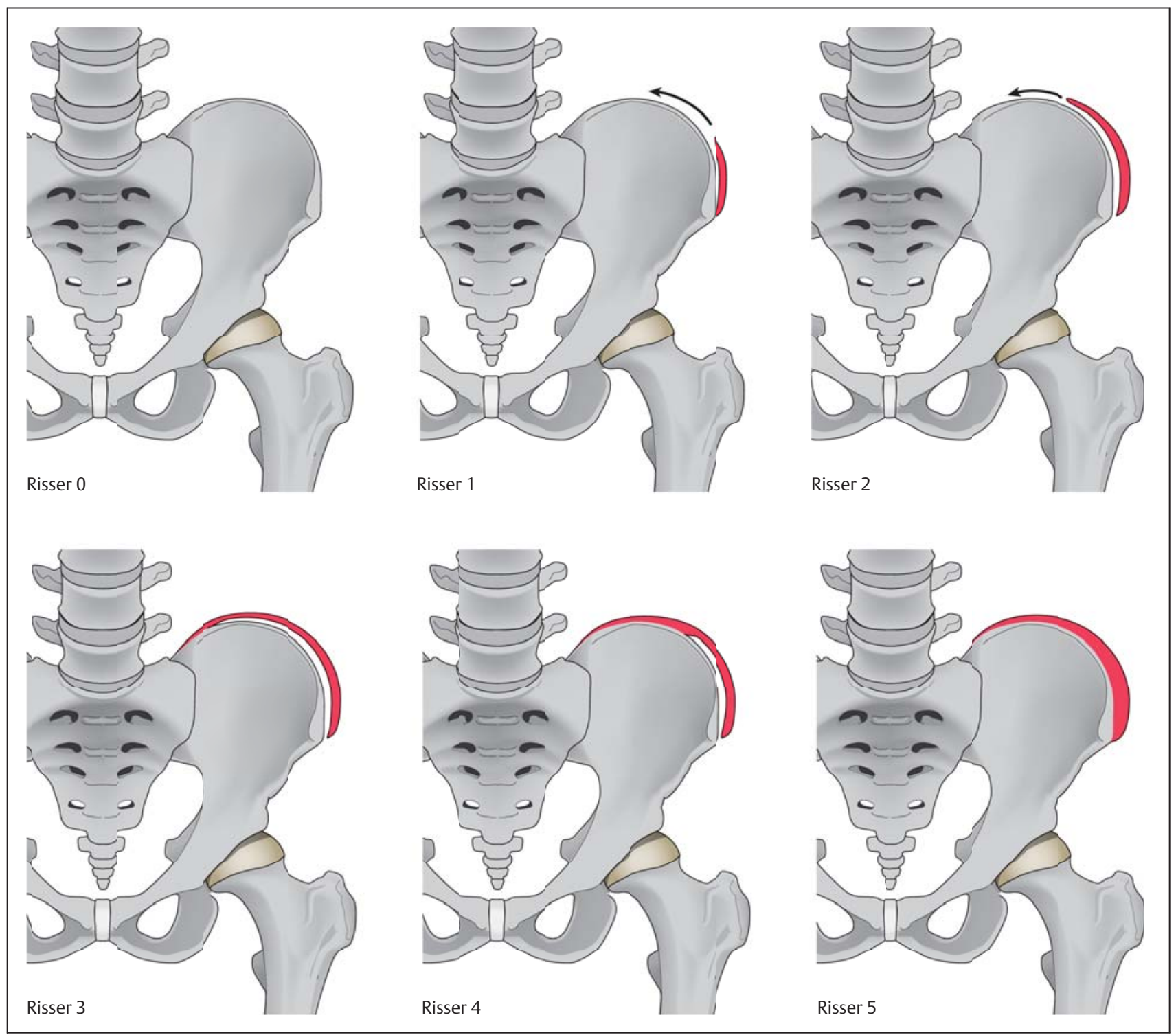

Abb. 5 - Risser-Stadien. Risser 0: Beckenkammapophyse noch nicht verknöchert. Risser I: Beckenkammapophyse am äußeren Rand der Beckenschaufel. Risser II: Beckenkammapophyse auf 50\% der Beckenschaufel. Risser III: Beckenkammapophyse entlang der gesamten Beckenschaufel sichtbar und bereits dorsal mit dem Ilium fusioniert. Risser IV: Beckenkammapophyse hälftig mit dem llium fusioniert. Risser V: vollständig verknöcherte Apophyse, die Darmbeinkammapophyse ist mit dem llium fusioniert.

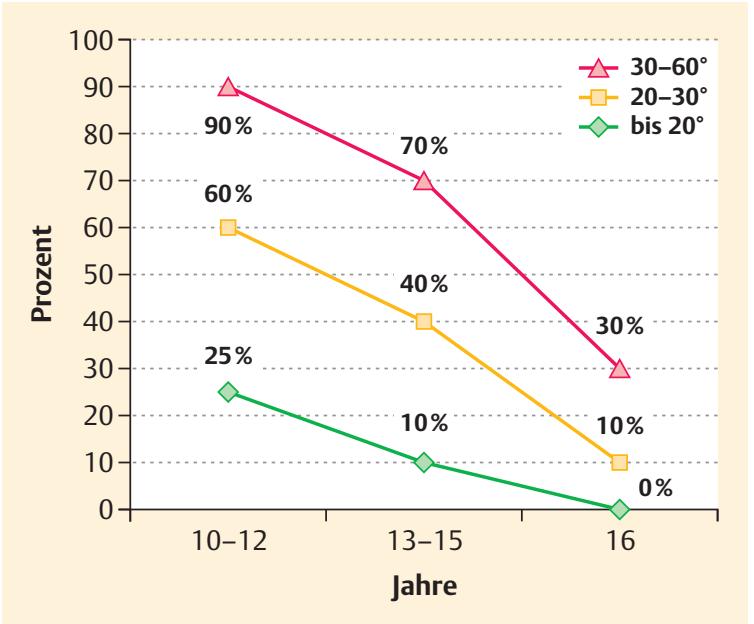

Abb. 6 - Progredienzverhalten der idiopathischen Adoleszentenskoliose im Wachstumsalter (nach [5]). Dargestellt ist das Verhalten unterschiedlicher Risikogruppen entsprechend des initialen COBB-Winkels. 


\section{Ganztägige Korsetttherapie}

\section{Prinzipien}

\section{Prinzip der Korsetttherapie}

Das Prinzip einer Korsettbehandlung besteht in der Wachstumslenkung der Deformität. Dazu muss ein passiv korrigierendes Rumpfkorsett auf eine noch wachsende Wirbelsäule treffen.
Die Indikation wird bei Skoliosen mit einem Winkel nach Cobb zwischen 20 und $40^{\circ}$ gesehen. Die Darmbeinkammapophyse sollte ein Reifezeichen nach Risser von weniger als III haben.

Es gibt 2 unterschiedliche Korsettanfertigungen:

- die individuelle Korsettanfertigung

- nach Gipsabdruck (häufigste Bauart) oder

- aufgrund einer computergestützten Oberflächenanalyse angefertigte Korsettanfertigung sowie

- die Veränderung von konfektionierten Körpern [10].

Der Gipsabdruck führt zum Formabdruck des deformierten Rumpfes. Daraus wird ein Positiv aus Gips gegossen und Flächen auf- bzw. abgetragen, um Freiräume oder Korrekturpelotten zu schaffen (Cheneau-Korsett). Darüber wird ein heißer Kunststoff gezogen, der mit Verstärkungen und Verschlüssen versehen wird.

Nach Anpassung und Eingewöhnung für den Patienten muss das Korsett 20-23 Stunden täglich getragen werden, was letztlich die Wachstumslenkung der Ringapophysen der Wirbel bewirken soll [11]. Um diese Korrekturwirkung nachweisen und Fehler am Korsett oder in der Anlage identifizieren zu können, sollte nach einigen Wochen eine erste Röntgenaufnahme als Wirbelsäulengesamtaufnahme im Stehen im Korsett ausgeführt werden. Hier wird eine Primärkorrektur von 30-40\% des Winkels vom Ausgangswinkel gefordert. Anderenfalls sind Compliance oder Korsettwirkung zu verbessern $[13,14]$. Für sportliche Aktivitäten soll das Korsett abgelegt werden, um die Verletzungsgefahr zu minimieren. Die Kinder erhalten zudem eine Teilsportbefreiung auf Ausdauer- und Sprungsportarten.

Bei der Ganztageskorsetttherapie ist eine tägliche Tragedauer von 23 Stunden erforderlich.

Das Korsett wird vom Patienten bis Wachstumsende (Risser-Stadium IV) getragen und dann unter forcierter
Physiotherapie abgelegt. Um die Compliance der Patienten zu erhalten, werden die in der Checkliste genannten Maßnahmen empfohlen [13].

Die Abschlussuntersuchung der Patienten erfolgt 2 Jahre nach Korsettablage mit Röntgen der Wirbelsäule. International wird eingeschätzt, dass mit einer Korsettbehandlung der Ausgangswinkel einer Skoliose zum Endergebnis gehalten ( $\pm 5^{\circ}$ Veränderung) oder leicht verbessert werden kann (mehr als $5^{\circ}$ Verbesserung zum Ausgangswert) [15].

Patienten mit Skoliosewinkeln von $>40^{\circ}$ sollten unbedingt in einem Skoliosezentrum bezüglich der Operationsindikation geprüft und nicht konservativ behandelt werden.

Die vorliegenden Langzeitergebnisse in Schweden belegen den Vorteil einer erfolgreichen Winkelkorrektur mit einer Operation im Vergleich zu nicht zufriedenstellenden Korsettergebnissen [16].

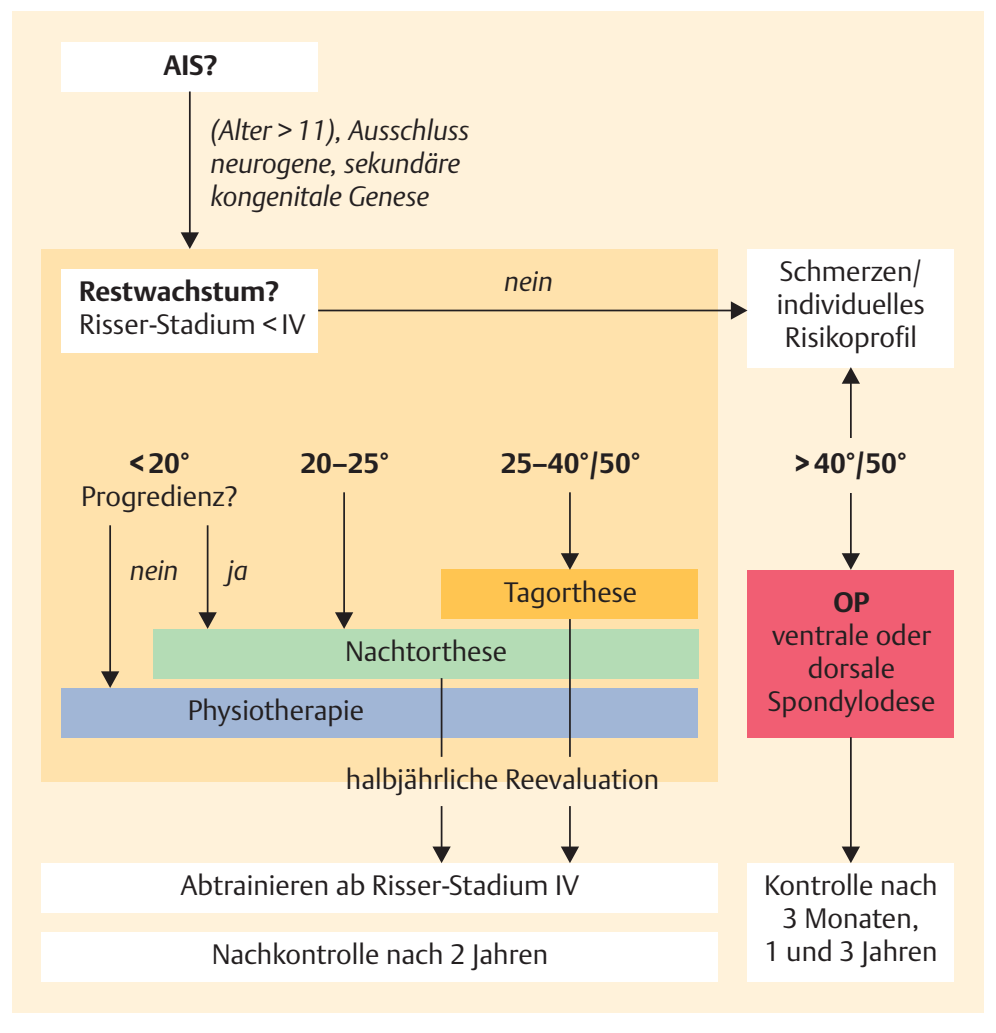

Abb. 7 - Dresdner Skoliosepfad. Die Entscheidung zur OP ist ab einem Cobb-Winkel von 40 (lumbal) $/ 50^{\circ}$ (thorakal) möglich. Handelt es sich jedoch um sehr junge Patienten mit hoher Wachstumsreserve, kann auch in diesem Winkelbereich ein Korsettversuch unternommen werden. 
Checkliste

\section{Begleitende Maßnahmen der Korsetttherapie}

regelmäßig:

Krankengymnastik des Rumpfes

\section{halbjährlich:}

Wiedervorstellungstermine

\section{jährlich:}

Röntgenkontrolle

Beurteilung der Orthese bezüglich Trageverhalten

mit resultierenden Gesprächen

2 Jahre nach Abschluss der Korsettbehandlung:

Abschlussuntersuchung

Röntgen

\section{- Sonderform nächtliche Korsettbehandlung}

Bereits in vergangenen Jahrzehnten wurden teilzeitige, nächtliche Orthesenbehandlungen etabliert [17]. Der breite Einsatz dieser Methode ohne Eingrenzung der Indikation führte jedoch zu signifikant schlechteren Ergebnissen im Bereich hoher Cobb-Winkelgrade. Erst die Eingrenzung auf einen geringeren Anfangswinkel zeigte ein gutes Behandlungsergebnis dieser Frühbehandlung mit Cobb-Winkeln von rund 20-25 $[17,18]$. Diese Methode ist gut geeignet, in frühen Erkrankungsphasen eine hohe Patientenakzeptanz und damit Compliance sowie technisch bedingt eine höhere Primärkorrektur mit Umkrümmungseffekten zu erreichen. Damit nehmen die positiv prädiktiven Faktoren Primärkorrektur und Compliance einen günstigen Einfluss auf das Outcome [19].

Um den Umkrümmungseffekt des Nachtkorsetts zusätzlich nutzen zu können, wird dieses im Dresdner Skoliosepfad zur Erhöhung der Primärkorrektur additiv zur Tagorthese in der ganztägigen Korsetttherapie eingesetzt (Abb. 7). Inwiefern durch den zusätzlichen Aufwand auch ein besseres Endergebnis entsteht, wird derzeit noch untersucht.

\section{Operative Therapie}

Oberhalb eines Skoliosewinkels nach Cobb von $40^{\circ}$ lumbal und $50^{\circ}$ thorakal wird empfohlen, die OP-Indikation zur Operation zu stellen. Winkelwerte darüber sind in Korsetten mechanisch nicht mehr zu beherrschen. Eine
Übersicht

\section{Zusammenfassung Korsetttherapie}

- Eine Korsetttherapie funktioniert nur am wachsenden Skelett in einem begrenzten Deformitätenausmaß (Cobb-Winkel 20-40 ).

- Compliance und Primärkorrektur sind wichtige Parameter des Erfolgs.

- Halbjährliche Wiedervorstellung der Patienten zur Kontrolle des Orthesensitzes und der Compliance sind wichtig.

spätere OP-Indikationsstellung führt zu Operationen mit größerer Morbidität und schlechterer Korrektur und Kosmetik.

Bei idiopathischen Skoliosen sollte ab einem CobbWinkel von $40-50^{\circ}$ eine OP-Indikation gestellt werden.

Der optimale Operationszeitpunkt liegt nach dem letzten Wachstumsschub, meist ca. 1 Jahr nach Menarcheeintritt oder zwischen dem 13. und 16. Lebensjahr. Sollte die operationsbedürftige Progredienz vorher eintreten, liegt eine prognostisch sogar noch ungünstigere juvenile Skoliose vor. Hier kann in speziellen Zentren wie folgt vorgegangen werden:

- verstärkte konservative Therapie mit Umkrümmungsgipsserien und Korsett,

- mitwachsende Systeme,

- kurzstreckige Korrekturspondylodese der Hauptkrümmung.

In den meisten Fällen, vor allem bei idiopathischer Skoliose, werden entsprechend der Klassifikation nach Lenke (Abb. 8) die Art der Krümmung, das lumbale Lotverhalten und das sagittale Profil bewertet und so die Länge und Zugangsweise der Skolioseoperation bestimmt [20]. Zur genauen Einordnung der Krümmungen in strukturelle und nichtstrukturelle Krümmungen sind Bending-Aufnahmen erforderlich.

Prinzipiell gibt es folgende Vorgehensweisen:

- ventrale Derotationsspondylodese (VDS)

- dorsale Korrekturspondylodese

- sowie die ventrodorsale kombinierte Technik in schwersten Fällen.

Die Morbidität der beiden Eingriffe ist gut untersucht und unterscheidet sich nicht wesentlich [21]. Es gibt 


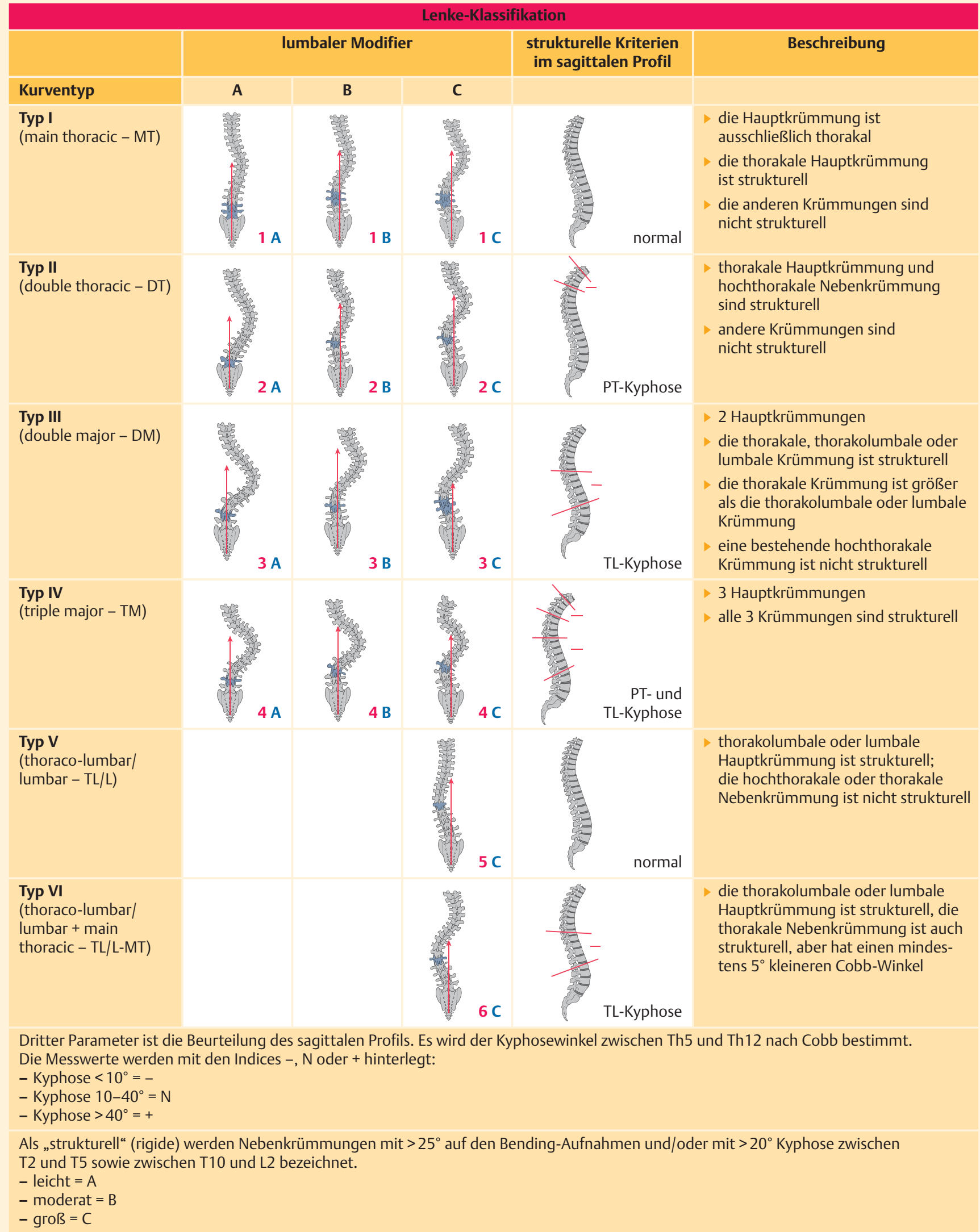

Abb. 8 - Lenke-Klassifikation (aus [6]). 


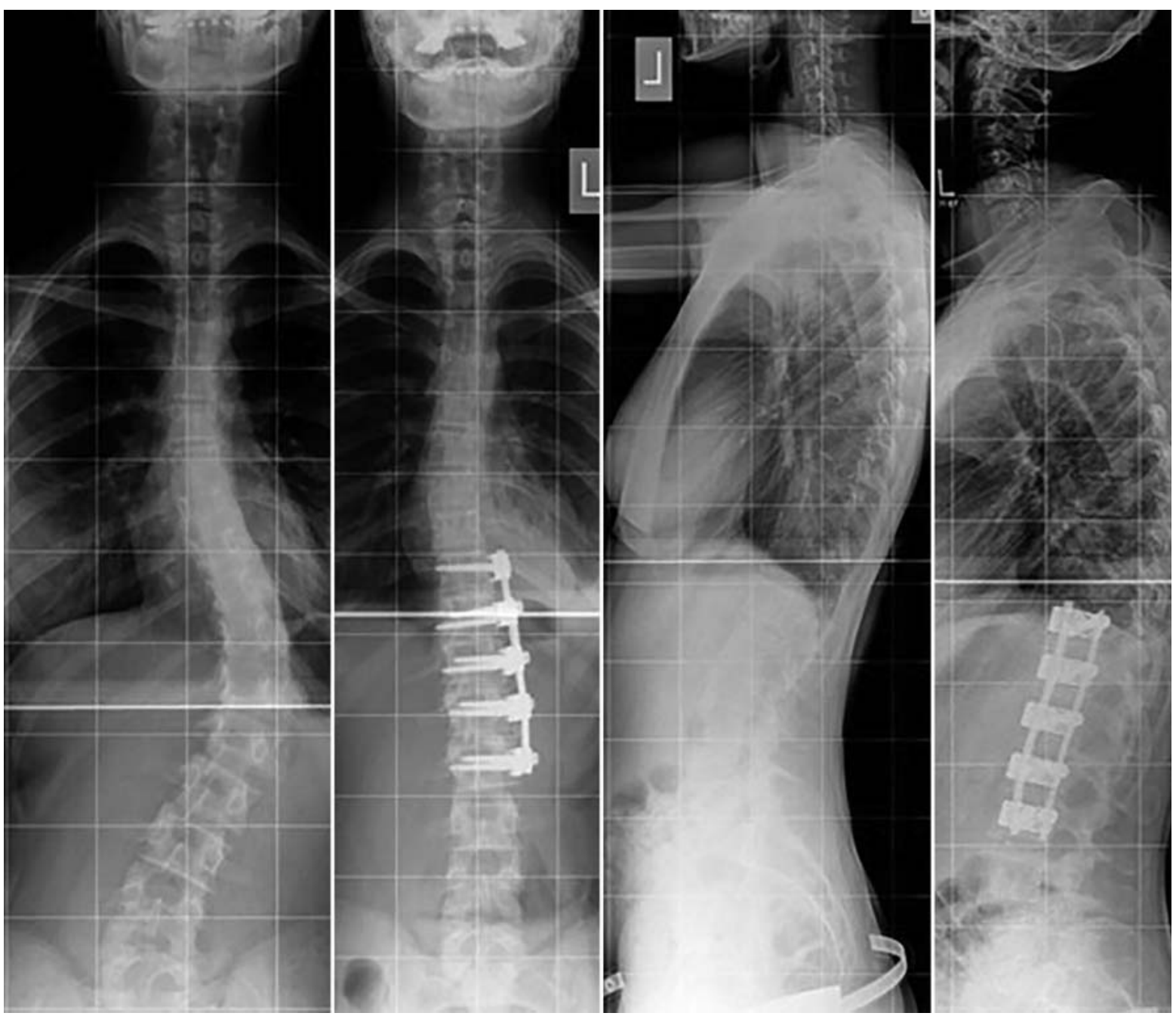

Abb. 9 - Doppelstab-VDS HZI (= Halm-Zielke-Instrumentation) im Bereich Th11 - L3. Komplette Rekonstruktion des Frontalprofils und Relordosierung im seitlichen Profil.

dagegen regionale, historische und personelle Unterschiede in der Indikationsstellung.

\section{- Ventrale Derotationsspondylodese (VDS)}

Anästhesiologisches Management. Die OP erfolgt unter spezialisierter Anästhesieführung, unter optimalen Voraussetzungen mit präoperativer PDK-Anlage (alternativ postoperative PCA), Wärme- und idealem Blutmanagement.

Zugang. Der Zugang besteht entweder bei Thorakalskoliose in einer Thorakotomie oder bei lumbalen und thorakolumbalen Indikationen in einer Thorakophrenolumbotomie.
OP-Technik. Nach konvexseitigem Freilegen der Wirbelsäule im Krümmungsbereich werden die Segmentgefäße ligiert und alle zu fusionierenden Bandscheiben entfernt. Nach Plattenapplikation und Verschraubung wird mittels zweier Korrekturstäbe (bei kleineren Wirbeln mitunter auch nur eines Stabes) eine Kompression der Konvexizität und eine Derotation über einen zumeist vorgebogenen, dickeren Augmentationsstab appliziert. In die Intervertebralräume werden Rippenstücke oder Cages eingebracht, um eine Kyphosierung des lumbalen Anteils zu vermeiden.

- Wichtig ist die optimale, planmäßige Korrektur der Deformität in frontaler und seitlicher Ebene (s. Abb. 9).

Prinzipiell ist eine ventrale Skolioseoperation auch minimalinvasiv, unter Verwendung von thorakoskopischer Technik möglich. 


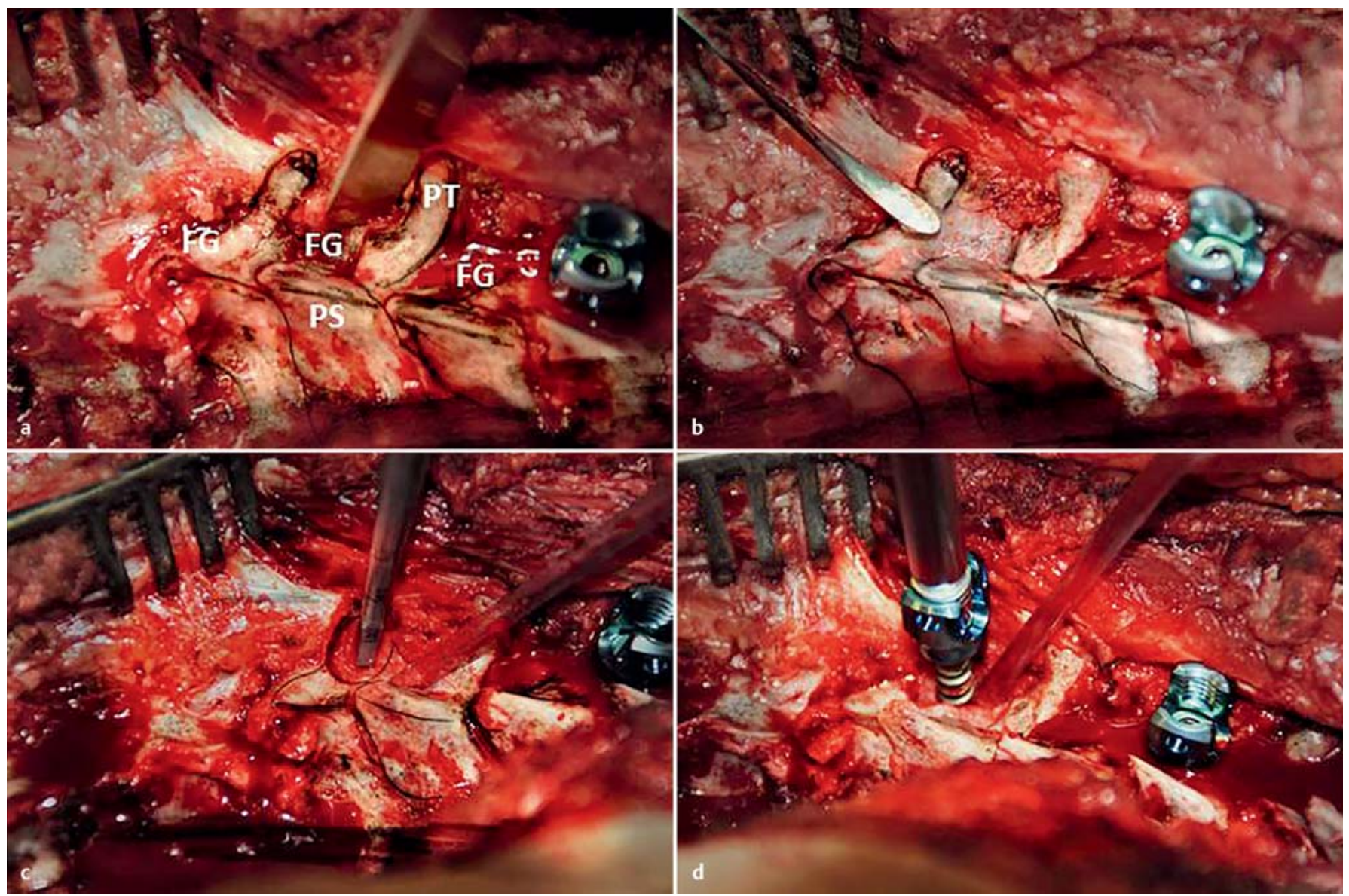

Abb. 10 - Pedikelschraubenapplikation in Freihandtechnik. a Aufmeißeln des Facettengelenks (FG). b Darstellen des unteren Gelenkpartners. c Priemen, Gewindeschnitt und Austasten. d Platzieren der Schraube. PS: Processus spinosus, PT: Processus transversus.

OP-Risiken/Komplikationen. Risiken der Operation bestehen im Verletzen der inneren Organe (Lunge, Darm, Milz, Niere) und der großen Gefäße, in der Fehlplatzierung der Schrauben und dem theoretischen Risiko eines vaskulären Querschnitts bei Ligatur der A. radicularis magna.

Folgebehandlung. Nach kurzzeitiger Pleuradrainage ist die korsettfreie Sofortmobilisation unter voller Belastung möglich (bei Ein-Stab-Systemen und/oder schlechter Knochenqualität ist mitunter noch ein Korsett erforderlich).

\section{- Dorsale Korrekturspondylodese}

Anästhesiologisches Management. Auch hierbei erfolgt eine entsprechend schonende anästhesiologische Führung, aber die Anlage des PDK zur Schmerztherapie am Ende der Operation in offener Technik. Zur Reduktion des Fremdblutbedarfs wird in der Operation ein Cell-Saver verwendet und das Blut unabhängig von der gesammelten Menge am Ende retransfundiert.
Zugang und OP-Technik. Nach dem dorsalen Zugang zur Wirbelsäule im Fusionssektor werden alle Wirbelgelenke aufgemeißelt. Dadurch wird es möglich, in Freihandtechnik Pedikelschrauben zu platzieren (Abb. 10; Film mit Echtzeitdarstellung der Freihandtechnik s. Abb. 11) [22]. Hierbei wird am Übergang vom lateralen zum mittleren Drittel des oberen Gelenkfortsatzes unter Aufnahme des Lotes in den eröffneten Processus transversus eingegangen. Diese Senkrechte führt in die optimale Pedikelachse entsprechend Rotation und Kyphose. Durch die Verwendung dieser Technik lässt sich sowohl Röntgen- als auch OP-Zeit deutlich einsparen.

Bei Problemen und sehr kleinen Pedikeln wird die Bildverstärkerunterstützung genutzt. Eine Alternative dazu wäre die Verwendung navigierter Systeme.

Nach Implantation von stabilen Pedikelschrauben an strategischen Regionen (in den Neutralwirbeln und im Scheitel) erfolgt die Skoliosekorrektur mit den in der Infobox „OP-Schritte und Tricks“ dargestellten Manövern. 
OP-Schritte und Tricks"

\section{Manöver zur intraoperativen Skoliosekorrektur}

\section{Cotrel-Debusset-Prinzip}

Rotation vorgebogener Stäbe aus Skoliose in Kyphose/Lordose.

\section{Harrington-Prinzip}

Bevorzugt Kompression konvexseitig und ggf. milde konkavseitige Distraktion.

\section{Translation}

Über Langkopfschrauben im Krümmungsscheitel oder mittels sublaminärer Bänder oder Drähte.

Apikale Derotation am Krümmungsscheitel unter Reduktion des Rippenbuckels mit speziellen Schrauben und Instrumenten.

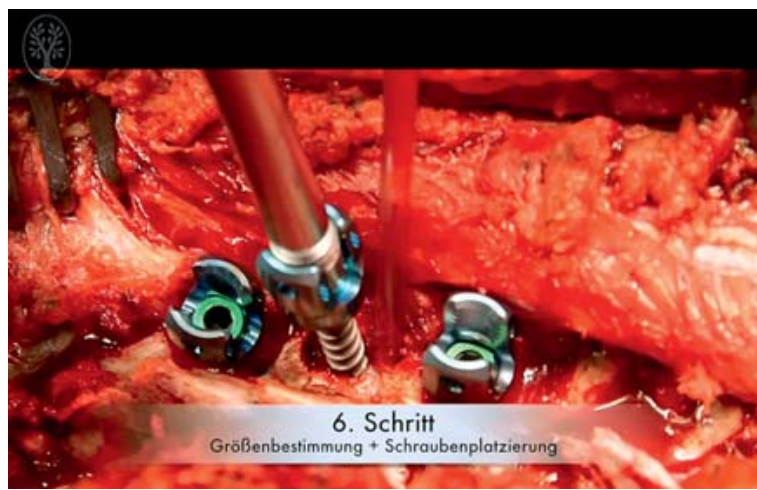

Abb. 11 - Echtzeitdarstellung der Freihandtechnik zur Platzierung der Pedikelschrauben im Rahmen der dorsalen Korrekturspondylodese. G $>$ B Video 1.

Wichtige Komponenten neben der Wiederaufrichtung der Seitabweichung sind

- die Horizontalisierung der Schultern am oberen Krümmungsende und

- die Remodellierung des seitlichen Profils durch gute Mobilisation der Wirbelsäule, Implantatverankerung und starre CrCo-Stäbe (s.Abb. 12).

Nach Durchführung der Korrektur sollte eine elektrophysiologische Kontrolle, alternativ ein Aufwachtest durchgeführt werden. Es konnte gezeigt werden, dass bei einer Inzidenz von 2,2\% auffälliger Befunde ein intraoperatives Neuromonitoring die Rate permanenter neurologischer Defizite auf 0,17\% senken konnte [23].

Zum Ende wird mittels einer Dekortikation der Wirbelsäule nach Risser die knöcherne Spondylodese angeregt. Das Einbringen von Beckenkammspongiosa, Fremdknochen oder Osteoinduktoren ist nicht erforderlich.
Die sorgfältige Dekortikation ist ein wichtiger Parameter für den Erfolg der Operation.

Folgebehandlung. Auch nach diesem Eingriff wird die korsettfreie Sofortmobilisation der Patienten und rasche Wiederaufnahme des alltäglichen Lebens angestrebt.

OP-Risiken/Komplikationen. Risiken der dorsalen Skolioseaufrichtung bestehen im Setzen eines neurologischen Schadens durch Schraubenfehlplatzierung oder durch zu starke Distraktion. Durch die Korrekturmanöver kann ein Flachrücken entstehen. Sehr starre Implantate wie CrCoStäbe erhöhen zwar die Korrekturwirkung, können aber gleichzeitig zum Ausreißen der Schrauben führen. Bei Beachtung der Instrumentierungsregeln und penibler Durchführung der dorsalen Spondylodese ist das Pseudarthroserisiko sehr gering.

\section{Postoperatives Management}

Durch das Verwenden eines PDK und durch intraoperative Wärmezufuhr können sowohl die Morbidität als auch die Krankenhausaufenthaltsdauer deutlich gesenkt werden.

Unmittelbar nach dem Eingriff werden die Kinder für eine Nacht intensivmedizinisch überwacht. Insbesondere distraktionsbedingte neurologische Störungen manifestieren sich erst am Abend des Operationstages, sodass eine kontinuierliche neurologische Überwachung erforderlich ist. Aufgrund der offenen Spondylodesestrecke bleibt die eingelegte Drainage am Anfang abgeklemmt und wird nur kurzzeitig intermittierend geöffnet.

In der Regel wird eine primärstabile Instrumentierung angestrebt, sodass die Kinder bereits ab dem 1. Tag voll mobilisierbar sind. Unter der Schmerztherapie des PDK soll bereits am 1. postoperativen Tag eine Mobilisation in den Sitz und spätestens ab dem 2. postoperativen Tag eine Mobilisation in den Stand möglich sein.

Zur Senkung des Infektrisikos wird der PDK am 5. oder 6. postoperativen Tag entfernt. Die Kinder verlassen in der Regel nach einer Woche das Krankenhaus und benötigen nur sporadisch eine NSAID-Schmerzmedikation.

Der Schulbesuch ist nach ca. 4 Wochen wieder möglich, die sportliche Betätigung kann nach dem ersten postoperativen Jahr langsam wieder gesteigert werden. Es erfolgen Wiedervorstellungen nach 3 Monaten, 1 und 3 Jahren postoperativ mit jeweiligen Röntgenkontrollen in 2 Ebenen. 

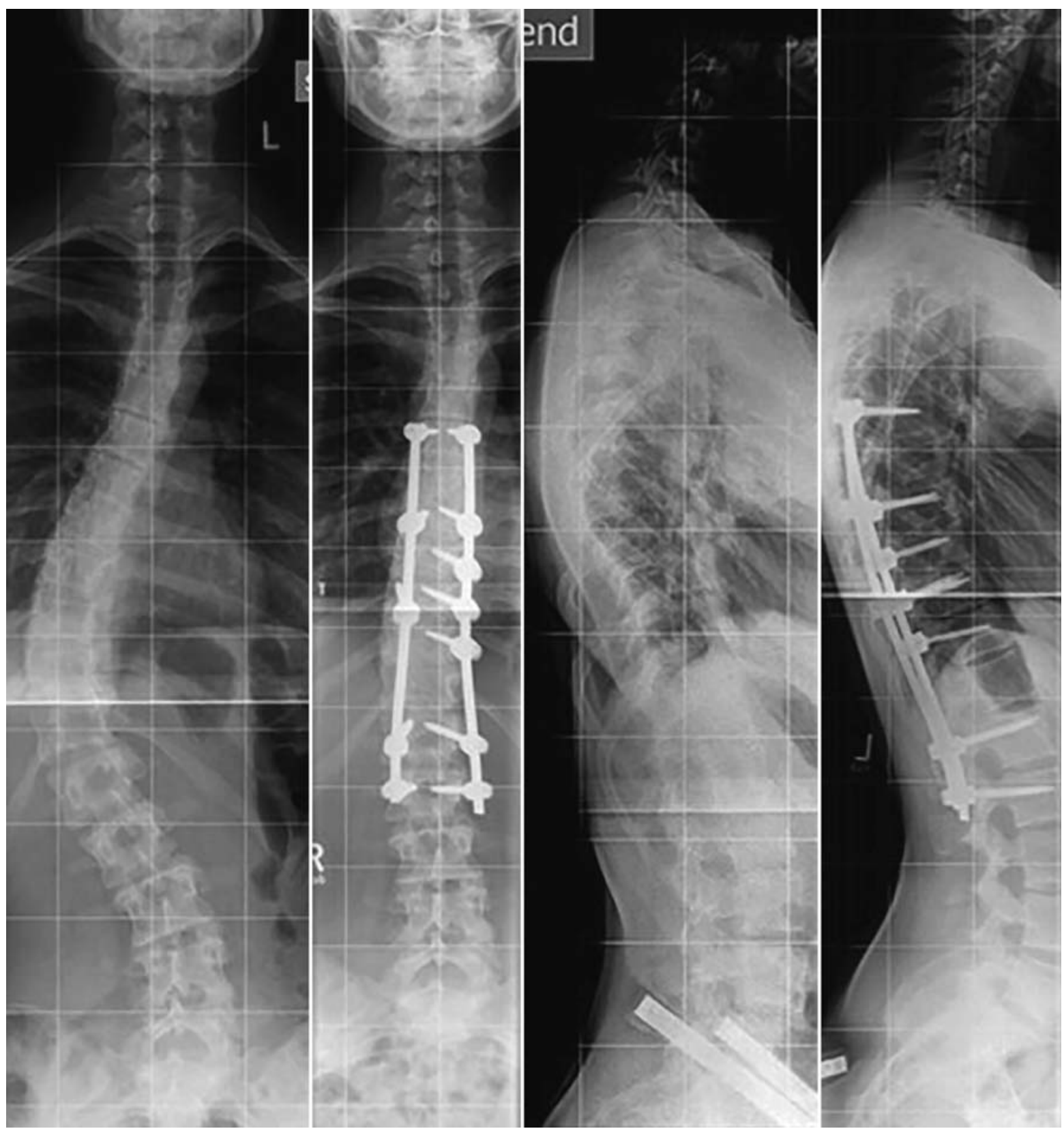

Abb. 12 - Dorsale Korrekturspondylodese Th6-L2.

\section{Begutachtung}

In Abhängigkeit des Krümmungsausmaßes wird bei Skoliosen von $30-60^{\circ}$ Cobb-Winkel ein GdB von $10-30 \%$, darüber hinaus auch bis $70 \%$ zuerkannt. Langstreckige Spondylodesen werden in Abhängigkeit der Fusionslänge, der statischen Dekompensation und der Einschränkung der Vitalkapazität zwischen 40-60\%, in Einzelfällen mit bis zu $80 \%$ GdB bewertet.
Für die berufliche Beratung sollen die Neigungen des Kindes berücksichtigt werden. Wir raten bei höhergradigen Skoliosen in der Regel von einem körperlich belastenden Beruf ab.

Interessenkonflikt: Die Autoren bestätigen, dass kein Interessenkonflikt vorliegt. 


\section{Video 1

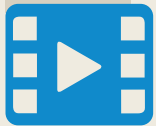

Das Video zeigt die intraoperative Anlage einer Feinnadelkatheterjejunostomie.

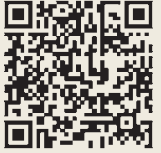

Das Video zu diesem Beitrag finden Sie im Internet unter http://dx.doi.org/10.1055/ s-0041-101843

\section{Korrespondenzadresse}

PD Dr. med. Peter Bernstein

UniversitätsCentrum für Orthopädie und Unfallchirurgie, Universitätsklinikum Carl Gustav Carus

Fetscherstr. 74

01307 Dresden

E-Mail: peter.bernstein@uniklinikum-dresden.de

\section{Literatur}

1 Cobb JR. The American Academy of orthopedic Surgeons instructional Course Lectures. Vol. 5. Ann Arbor, MI: Edwards; 1948

2 Miller F. Cerebral Palsy. In: Yazici M, ed. Non-idiopathic Spine Deformities in young Children. Berlin: Springer; 2011: 77-86

3 Schlenzka D. Duchenne's muscular Dystrophy and spinal muscular Atrophy. In: Yazici M, ed. Non-idiopathic Spine Deformities in young Children. Berlin, Heidelberg: Springer; 2011: 87-95

4 Konieczny MR, Senyurt H, Krauspe R. Epidemiology of adolescent idiopathic scoliosis. J Child Orthop 2013; 7: 3-9

5 Nachemson A, Sahlstrand T. Etiologic factors in adolescent idiopathic scoliosis. Spine 1977; 2: 176-184

6 Stücker P. Die idiopathische Skoliose. Orthop Unfallchir up2date 2010; 5: $39-56$

7 Burwell RG, Dangerfield PH, Moulton A et al. Whither the etiopathogenesis (and scoliogeny) of adolescent idiopathic scoliosis? Incorporating presentations on scoliogeny at the 2012 IRSSD and SRS meetings. Scoliosis 2013; 8: 4

8 Hefti F. Pathogenesis and biomechanics of adolescent idiopathic scoliosis (AIS). J Child Orthop 2013; 7: 17-24

9 Danielsson AJ. Natural history of adolescent idiopathic scoliosis: a tool for guidance in decision of surgery of curves above 50 degrees. J Child Orthop 2013; 7: 37-41

10 Schaal A, Cheneau J. Lage, Wirkung und Form der Druckzonen im Cheneau-Korsett. Orthopädie-Technik 1995; 4: 317-321

11 Niethard FU. Kinderorthopädie. Stuttgart, New York: Thieme; 2010

12 Rowe DE, Bernstein SM, Riddick MF et al. A meta-analysis of the efficacy of non-operative treatments for idiopathic scoliosis. J Bone Joint Surg Am 1997; 79: 664-674

13 Landauer F, Wimmer C, Behensky H. Estimating the final outcome of brace treatment for idiopathic thoracic scoliosis at 6-month followup. Pediatr Rehab 2003; 6: 201 - 207

14 Seifert J, Selle A, Flieger C et al. Die Compliance als Prognosefaktor bei der konservativen Behandlung idiopathischer Skoliosen. Orthopäde 2009; 38: 151 - 158

15 Richards BS, Bernstein RM, D’Amato CR et al. Standardization of criteria for adolescent idiopathic scoliosis brace studies: SRS Committee on Bracing and Nonoperative Management. Spine (Phila Pa 1976) 2005; 30: 2068 -2075; discussion 2076-2077

16 Danielsson AJ, Nachemson AL. Radiologic findings and curve progression 22 years after treatment for adolescent idiopathic scoliosis: comparison of brace and surgical treatment with matching control group of straight individuals. Spine 2001; 26: 516 - 525

17 Gepstein R, Leitner Y, Zohar E et al. Effectiveness of the Charleston bending brace in the treatment of single-curve idiopathic scoliosis. J Pediatr Orthop 2002; 22: 84-87

18 Seifert ], Selle A. [Is night-time bracing still appropriate in the treatment of idiopathic scoliosis?] Orthopäde 2009; 38: 146-150

19 Vijvermans V, Fabry G, Nijs J. Factors determining the final outcome of treatment of idiopathic scoliosis with the Boston brace: a longitudinal study. J Pediatr Orthop B 2004; 13: 143 - 149

20 Lenke LG, Edwards CC, Bridwell KH. The Lenke classification of adolescent idiopathic scoliosis: How it organizes curve patterns as a template to perform selective fusions of the spine. Spine (Phila Pa 1976) 2003; 28 (20S): S199-S207

21 Geck MJ, Rinella A, Hawthorne D et al. Comparison of surgical treatment in Lenke 5C adolescent idiopathic scoliosis: anterior dual rod versus posterior pedicle fixation surgery: a comparison of two practices. Spine (Phila Pa 1976) 2009; 34: 1942 - 1951

22 Chung KJ, Suh SW, Desai S et al. Ideal entry point for the thoracic pedicle screw during the free hand technique. Int Orthop 2008; 32: $657-662$

23 Thuet ED, Winscher JC, Padberg AM et al. Validity and reliability of intraoperative monitoring in pediatric spinal deformity surgery: a 23 year experience of 3436 surgical cases. Spine (Phila Pa 1976) 2010; 35: $1880-1886$ 


\section{CME-Fragen}

\section{CME•thieme.de}

\section{CME-Teilnahme}

- Viel Erfolg bei Ihrer CME-Teilnahme unter http://cme.thieme.de

- Bitte informieren Sie sich vorab online über die Gültigkeitsdauer.

- Sollten Sie Fragen zur Online-Teilnahme haben, unter http://cme.thieme.de/hilfe finden Sie eine ausführliche Anleitung.

Welches ist kein klinisches Zeichen einer Skoliose?
A Asymmetrische Taillendreiecke
B Schulterhochstand
C Tannenbaumphänomen
D Lotüberhang
E Rippenbuckel

Welche Aussage zur Klinik bzw. Diagnostik von Skoliosen trifft zu?
A Die Skoliose ist per se eine schmerzarme/schmerzfreie Erkrankung.

B Zur Erstdiagnostik jeder Skoliose gehört eine Kernspintomografie der gesamten Wirbelsäule.

C Bei gesunden Kindern wird die Röntgenaufnahme der gesamten Wirbelsäule in der Regel im Sitzen angefertigt.

D Neuromyopathische Skoliosen weisen fast immer eine ausgeprägte Rotationskomponente auf.

E Mit dem Eintritt der Menarche kann sich die Skoliose nicht weiter verschlechtern.
Welche Form der Wirbelmissbildung weist das höchste Progredienzrisiko auf?
A Schmetterlingswirbel

B Lateral Bar und kontralateraler Halbwirbel

C gegenüberliegende Halbwirbel

D isolierte Halbwirbel

E Keilwirbel
Eine der folgenden Aussagen zu neuromuskulären Skoliosen trifft nicht zu. Welche?
A Neuromuskuläre Skoliosen sind zu einem hohen Prozentsatz progredient.

B Bei Progredienz sollte eine neuromuskuläre Skoliose frühzeitig einer operativen Versorgung zugeführt werden.

c Versteifende Operationen sollten erst ab dem 10. Lebensjahr durchgeführt werden.

D Versteifende Operationen erfordern meistens ein langstreckiges Vorgehen bis LWK 5.

E Eine Korsettbehandlung neuromuskulärer Skoliosen ist immer sinnlos. 


\section{Wirbelsäule}

Welche Aussage hinsichtlich Skoliosen trifft zu?
A Juvenile Skoliosen sind harmlose Varianten der Adoleszentenskoliosen.

B Skoliosen bei Neurofibromatose sind in der Regel langbogig C-förmig.

C Schwere Adoleszentenskoliosen treten häufiger bei Mädchen auf.

D Skoliosen bei Jungen verlaufen häufig schwerer.

E Bei myopathischen Skoliosen sollte die OP-Indikation noch im Alter der erhaltenen Gehfähigkeit gestellt werden.
Eine der folgenden Aussagen zum natürlichen Verlauf der idiopathischen Skoliose trifft nicht zu. Welche?
A Bei etwa $3 / 4$ der skelettal unreifen Patienten mit einem deutlich sichtbaren Rippenbuckel wird die idiopathische Skoliose einen progredienten Verlauf nehmen.

B Ab dem Risser-Stadium IV ist das Risiko einer Progredienz minimal.

C Je stärker die Krümmung, desto höher ist das Risiko einer Progredienz.

D Unbehandelte idiopathische Adoleszentenskoliosen sind häufig mit einer erhöhten Mortalität assoziiert.

E Bei Skoliosen ab einem Cobb-Winkel von $50^{\circ}$ ist auch im Erwachsenenalter mit einer Progredienz zu rechnen.
Was ist kein Charakteristikum der Korsetttherapie?
A Die Primärkorrektur ist entscheidend für das Endergebnis.

B Die Compliance spielt bei Jugendlichen in der Pubertät keine besondere Rolle.

C Bei der ganztägigen Korsetttherapie ist eine Tragedauer von 20-23 Stunden erforderlich.

D Eine fortwährende Begleitung der Patienten in halbjährlichen Abständen ist anzustreben.

E Mit dem Nachtkorsett kann eine Umkrümmung erreicht werden.
Für die Durchführung von Skolioseoperationen erforderlich ...
A ... ist eine mit dem operativen Ablauf vertraute Anästhesie.

B ... ist die intraoperative Wärmezufuhr.

C ... ist die postoperative Schmerztherapie, idealerweise mittels PDK.

D ... ist ein optimales Blutungsmanagement.

E ... sind alle unter A-D genannten Faktoren.
Eine der folgenden Aussagen zur dorsalen Korrekturspondylodese ist falsch. Welche?
A Die Freihandtechnik ermöglicht das Auffinden des Pedikels anhand definierter anatomischer Landmarken.

B CrCo-Stäbe sind weich und schmiegen sich den natürlichen Kurven des Patienten hervorragend an.

C Über die apikale Derotation kann eine Reduktion des Rippenbuckels erreicht werden.

D Nach Durchführung der Skoliosekorrektur sollte eine neurologische Kontrolle, z.B. durch einen intraoperativen Aufwachtest, erfolgen.

E Eine sorgfältige Spondylodesetechnik ist wichtig zur Vermeidung von Pseudarthrosen.
Wodurch ist die postoperative Situation nach Skolioseoperation gekennzeichnet?
A Drainagen müssen kontinuierlich auf Sog bleiben.

B Die Mobilisation sollte erst nach einer Woche begonnen werden.

C Die Schule kann erst ein halbes Jahr nach Operation wieder besucht werden.

D Körperlich belastende Berufe sind für Patienten nach Skolioseoperation eher ungeeignet.

E Nach Skolioseoperation wird immer ein GdB von > 50\% zuerkannt. 